\title{
Rational maps and string topology
}

\author{
SADOK KALLEL \\ PAOLO SALVATORE
}

\begin{abstract}
We apply a version of the Chas-Sullivan-Cohen-Jones product on the higher loop homology of a manifold in order to compute the homology of the spaces of continuous and holomorphic maps of the Riemann sphere into a complex projective space. This product makes sense on the homology of maps from a co- $H$ space to a manifold, and comes from a ring spectrum. We also build a holomorphic version of the product for maps of the Riemann sphere into homogeneous spaces. In the continuous case we define a related module structure on the homology of maps from a mapping cone into a manifold, and then describe a spectral sequence that can compute it. As a consequence we deduce a periodicity and dichotomy theorem when the source is a compact Riemann surface and the target is a complex projective space.
\end{abstract}

58D15; 55R20, 26C15

\section{Introduction}

Spaces of maps, holomorphic and continuous, from a Riemann surface into complex projective spaces and more generally flag manifolds have been much studied in recent years. These are spaces that occur naturally in physics (sigma-models, topological field theory and gauge theory), in engineering (control theory) and also in the theory of minimal surfaces and harmonic maps. Our main interest in this paper and in its sequel [16] is the topology and in particular the homology of these spaces.

One main interest of the present work is that it gives the first instance where the intersection product techniques of the newly developed theory of "string topology" (Chas-Sullivan and Cohen-Jones $[2 ; 7]$ ) are used to give complete understanding of the homology of certain mapping spaces. In terms of specific computations, this paper determines the homology of maps of a two sphere into complex projective space. It then derives important consequences for the homology of spaces of maps from a more general closed Riemann surface. It further adapts the "string" techniques, mainly as developed by Cohen and Jones, to the holomorphic setting and computes the homology of the subspace of holomorphic maps. Without use of the intersection 
products introduced originally by Chas and Sullivan, these computations would be hard to access.

In [2], Chas and Sullivan introduced new structures on the homology of parameterized and unparameterized loop spaces of closed manifolds. One main observation there was the existence of a product on the (regraded) homology of the free loop space of a closed manifold which was compatible via evaluation at a basepoint with the intersection product on the homology of the manifold in question. In [7], J D S Jones and R Cohen gave a homotopy theoretic and more rigorous definition of a similar product $\bullet$ and asserted it was the same as Chas and Sullivan's. Shortly after and jointly with J Yan [8] they showed that $\bullet$ did fit naturally in the Serre spectral sequence of the evaluation fibration to yield a multiplicative second quadrant spectral sequence (or loop spectral sequence) which allows explicit computations. Further interesting extrapolations on this spectral sequence have been introduced recently by Le Borgne [19].

Our work exploits these ideas in various ways, it expands on them to pass from ring to module structures and then adapts them to the holomorphic category. It also records the fact that the results of [7] and hence presumably [2] need not restrict to maps from the circle but extend naturally to maps from coassociative co- $H$ spaces.

The theorem next and its corollary expand on the work of Cohen and Jones. The fruitful idea in [7] was to combine pullback diagrams of Hilbert manifolds and Thom-Pontrjagin collapse maps. Write $\operatorname{Map}(X, Y)$ for the space of continuous maps from $X$ into $Y$ and denote by $e v: \operatorname{Map}(X, Y) \longrightarrow Y$ the evaluation map $e v(f):=f\left(x_{0}\right)$ where $x_{0} \in X$ is a basepoint. For $M$ a closed compact (smooth) manifold of dimension $d$, let $T M$ be its tangent bundle. For $X$ a (based) topological space, one can define a Thom spectrum $\operatorname{Map}(X, M)^{-T M}$ as follows. Let $v$ be the normal bundle of an embedding $M \rightarrow \mathbb{R}^{\ell}$, and $e v^{*}(v)$ its pullback over $\operatorname{Map}(X, M)$ via evaluation. One then defines $\operatorname{Map}(X, M)^{-T M}$ to be the $\ell$-fold desuspension of the suspension spectrum of the Thom space of $e v^{*}(v)$. When $X=*$, then $M^{-T M}$ is Spanier-Whitehead dual to $M_{+}$by a well known result of Atiyah.

All spaces are assumed to be path-connected and compactly generated.

Theorem 1.1 Suppose $S$ is a coassociative (cocommutative) co- $H$ space coacting on a space $X$. Then

(1) $\operatorname{Map}(S, M)^{-T M}$ is a (homotopy commutative) ring spectrum;

(2) $\operatorname{Map}(X, M)^{-T M}$ is a module over the ring spectrum $\operatorname{Map}(S, M)^{-T M}$.

This theorem as it turns out is a consequence of a more general statement. Let $X_{1}, X_{2}$ be based topological spaces and suppose $M$ is a closed manifold. Then there is a 
pairing of spectra

(1-1) $\quad \operatorname{Map}\left(X_{1}, M\right)^{-T M} \wedge \operatorname{Map}\left(X_{2}, M\right)^{-T M} \longrightarrow \operatorname{Map}\left(X_{1} \vee X_{2}, M\right)^{-T M}$

with relevant associativity properties and commuting via the evaluation maps with the ring multiplication $M^{-T M} \wedge M^{-T M} \longrightarrow M^{-T M}$. Such a pairing exists because it is possible to construct a tubular neighborhood of $\operatorname{Map}\left(X_{1} \vee X_{2}, M\right)$ in $\operatorname{Map}\left(X_{1}, M\right) \times$ $\operatorname{Map}\left(X_{2}, M\right)$, strictly compatible with evaluation fibrations and living over the normal neighborhood of the diagonal $M \hookrightarrow M \times M$ (Lemma 3.1). Part (1) of Theorem 1.1 for suspensions has also been obtained by Klein in [17] using a different approach.

Most important for us is Theorem 1.1 (2) in the case when $S$ is the $n$-sphere $(n \geq 1)$, $X$ an $n$-manifold and the coaction map $X \longrightarrow X \vee S$ is obtained by pinching the boundary of a small embedded disk in $X$. The following corollary is key to our homological calculations.

Corollary 1.2 Let $N, M$ be two closed compact oriented manifolds of dimension $n$ and $d$ respectively. Then $\mathbb{H}_{*}\left(L^{n} M\right)$ is a ring and $\mathbb{H}_{*}(\operatorname{Map}(N, M))$ is a module over $\mathbb{H}_{*}\left(L^{n} M\right)$, where $\mathbb{H}_{*}=H_{*+d}$ and $L^{n} M=\operatorname{Map}\left(S^{n}, M\right)$. This module structure is compatible via the evaluation maps with the intersection product on $\mathbb{H}_{*}(M)$.

Note that orientability is needed here but not in earlier statements. The ring multiplication above (which we dub simply intersection product) reduces to the Cohen-Jones product for $n=1$. Such product has been investigated by Chataur [3] and $\mathrm{Hu}$ [13] as well. Note also that it is possible to replace singular homology in Corollary 1.2 by other multiplicative generalized homology theories in line with work in Cohen and Godin [6] (see Section 3). It is equally possible to expand on Theorem 1.1 (1) by considering bundles over $M$ with fibrewise multiplication hence obtaining further interesting pairings between spectra (Gruher and Salvatore [11]).

More interestingly perhaps from the physicist's point of view is to find out whether the module and ring structures described above can be carried out in the holomorphic category. Let $M$ be a complex algebraic variety and write $\operatorname{Hol}(C, M) \subset \operatorname{Map}(C, M)$ for the subspace of all holomorphic maps from a compact Riemann surface $C$ into $M$. When $C=\mathbb{P}^{1}$ is the Riemann sphere, we reduce this notation to $\operatorname{Hol}(M)$. This space for $M$ compact splits into a number of connected components depending on the geometry of $M$ and each component is of the homotopy type of a finite dimensional complex. It might happen of course that $\operatorname{Hol}(M)$ has no non-constant maps. However when $M=G / P$ is a generalized flag manifold, $G$ being a complex Lie group and $P$ a parabolic subgroup, $\operatorname{Hol}(G / P)$ has a rich topology (Boyer, Mann, Hurtubise, Milgram and Segal $[1 ; 20])$. We manage to show in Section 5 that an intersection product similar to the Cohen-Jones product exists on the (regraded) homology of the space $\operatorname{Hol}(G / P)$. 
Theorem 1.3 The Thom spectrum $\operatorname{Hol}(G / P)^{-T(G / P)}$ is a homotopy commutative ring spectrum. In particular $\mathbb{U}_{*}(\operatorname{Hol}(G / P))=H_{\operatorname{dim}_{\mathbb{R}}}(G / P)+*(\operatorname{Hol}(G / P))$ is a commutative graded ring.

In proving this theorem we adapt many of the constructions of Section 3 to the holomorphic category; in particular the existence and compatibility of tubular neighborhoods. The main geometric ingredient we use is a construction of Gravesen and Segal [10] which represents such holomorphic maps in terms of certain configuration spaces of principal parts (cf Section 5). In the case when $G / P=\mathbb{P}^{n}$ we further make the observation that the intersection products that we construct in Corollary 1.2 and Theorem 1.3 are compatible via subspace inclusion (Section 4).

Proposition 1.4 The inclusion $\operatorname{Hol}\left(\mathbb{P}^{n}\right) \hookrightarrow L^{2} \mathbb{P}^{n}$ induces a homomorphism of ring spectra $\operatorname{Hol}\left(\mathbb{P}^{n}\right)^{-T \mathbb{P}^{n}} \rightarrow\left(L^{2} \mathbb{P}^{n}\right)^{-T \mathbb{P}^{n}}$. In particular $\mathbb{H}_{*}\left(\operatorname{Hol}\left(\mathbb{P}^{n}\right)\right) \rightarrow \mathbb{H}_{*}\left(L^{2} \mathbb{P}^{n}\right)$ is a ring homomorphism.

We expect the ring structure uncovered by Theorem 1.3 to be highly instrumental in extending the homology calculations of [1] for based holomorphic maps to the unbased case. We verify that this is indeed the case for $G / P=\mathbb{P}^{n}$. Write for simplicity $\operatorname{Hol}(n):=\operatorname{Hol}\left(\mathbb{P}^{1}, \mathbb{P}^{n}\right)$ and as customary write $\operatorname{Rat}(n)$ for the subspace of based holomorphic maps $\mathbb{P}^{1} \rightarrow \mathbb{P}^{n}$. If $\Omega^{2}\left(\mathbb{P}^{n}\right)$ is the corresponding subspace of based continuous maps, then we have the diagram of inclusions

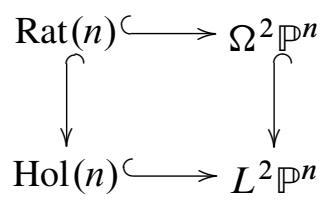

All connected components in this case are labeled by integers $k$ and we write Rat ${ }_{k} \subset$ $\mathrm{Hol}_{k} \subset L_{k}^{2}$ the corresponding components of degree $k$. In the holomorphic case $k$ is non-negative and is the algebraic degree, while in the continuous case it takes on all values. The intersection product turns out to be additive on degree.

In studying the Serre spectral sequence relating based and unbased mapping spaces in (1-2), standard properties and comparison methods are not enough to determine all differentials. To bypass this shortcoming, the basic idea has been then, using the fact that $\mathbb{P}^{n}$ is closed oriented, to concoct a regraded version of this Serre spectral sequence to get a multiplicative spectral sequence with respect to the intersection product $\bullet$ introduced earlier, and hence be able to decide about differentials using the fact that they are now derivations. This basic idea goes back to Cohen, Jones, and Yan [8] who worked out its technical details for the free loop space. In Section 6 we show that the 
techniques of [8] can be straightforwardly adapted to higher loop spaces eventhough the fibers are now non-connected. More explicitly we construct a second quadrant spectral sequence of algebras $E^{r}\left(S^{2}, \mathbb{P}^{n}\right)$ which converges to $\mathbb{H}_{*}\left(L^{2} \mathbb{P}^{n}\right)=H_{2 n+*}\left(L^{2} \mathbb{P}^{n}\right)$. This fact as it pleasantly turns out is sufficient to determine entirely $H_{*}\left(L^{2} \mathbb{P}^{n}\right)$ with field coefficients, and by standard comparison arguments determine $H_{*}(\mathrm{Hol}(n))$ as well. This computation is in fact at the origin of this work.

Let us recall the homology of the based holomorphic subspace as described by Cohen, Cohen, Mann and Milgram in [4]. It was there shown that Rat $(n)$ is a $C_{2}$-space (a space with an action of the little two-discs operad); in particular it is an $H$-space and the "forgetful" map $\operatorname{Rat}(n) \hookrightarrow \Omega^{2} \mathbb{P}^{n}$ is a $C_{2}$-map up to homotopy (and hence an $H$-map) inducing a homology monomorphism. We make the caution that this monomorphism is special to the genus 0 case and does not hold for maps from positive genus curves as is discussed by the first author in [15] for example.

At the prime 2, the results of [4] give an algebra isomorphism

$$
H_{*}\left(\operatorname{Rat}(n) ; \mathbb{Z}_{2}\right)=\mathbb{Z}_{2}\left[\iota, u, Q(u), Q^{2}(u), \ldots\right]
$$

where the algebra structure corresponds to the Pontrjagin product, and $\iota$ and $u$ are the images of the bottom and top homology generators of $S^{2 n-1}$ via a homotopy equivalence $S^{2 n-1} \simeq \operatorname{Rat}_{1}(n)$ (cf Section 2). Moreover $Q^{i}(u):=Q \cdots Q(u) \in$ $H_{2^{i+1} n-1}\left(\operatorname{Rat}_{2^{i}}(n) ; \mathbb{Z}_{2}\right)$ is the $i$-fold iterated first Dyer-Lashof operation on $u$. Now the homology of the continuous mapping space $\Omega^{2} \mathbb{P}^{n}$ is obtained from the homology of $\operatorname{Rat}(n)$ by inverting multiplicatively $\iota$, compatibly with the fact that $\Omega^{2} \mathbb{P}^{n}$ is the (topological) group completion of $\operatorname{Rat}(n)$.

With coefficients in $\mathbb{Z}_{p}$, with $p$ an odd prime, the statement is analogous, for we have

$$
H_{*}\left(\operatorname{Rat}(n) ; \mathbb{Z}_{p}\right)=E\left[u, Q(u), Q^{2}(u), \ldots\right] \otimes \mathbb{Z}_{p}\left[\iota, \beta Q u, \beta Q^{2} u, \ldots\right]
$$

where $\beta$ is the mod $-p$ homology Bockstein, while $H_{*}\left(\Omega^{2} \mathbb{P}^{n} ; \mathbb{Z}_{p}\right)$ is obtained by inverting $\iota$. Rationally the situation is trivial as all positive components of $\operatorname{Rat}(n)$ and all components of $\Omega^{2} \mathbb{P}^{n}$ are rationally equivalent to $S^{2 n-1}$ (cf Section 2). Note that multiplication by $\iota$ or $u$ switches components up by one.

To state our main computation, write $H^{*}\left(\mathbb{P}^{n}\right)=\mathbb{Z}[c] / c^{n+1}$ and grade it negatively so that $c \in H^{-2}\left(\mathbb{P}^{n}\right)$. Note that $H_{*}\left(\Omega^{2} \mathbb{P}^{n}\right) \otimes H^{*}\left(\mathbb{P}^{n}\right)$ is an algebra with product induced from the Pontrjagin product in the first factor and the cup product in the second factor. 


\section{Theorem 1.5}

(1) Continuous The homology $\mathbb{H}_{*}\left(L^{2} \mathbb{P}^{n}\right)$ with coefficients in a field is (additively) the homology of the differential graded algebra $H_{*}\left(\Omega^{2} \mathbb{P}^{n}\right) \otimes H^{*}\left(\mathbb{P}^{n}\right)$ with differential $d$ such that $d \iota=(n+1) u c^{n}$ and $d$ vanishes on all other generators.

(2) HOLOMORPHIC The homology $\mathbb{H}_{*}(\operatorname{Hol}(n))$ with coefficients in a field is (additively) the homology of the differential graded subalgebra $H_{*}(\operatorname{Rat}(n)) \otimes H^{*}\left(\mathbb{P}^{n}\right)$ with the same differential as above.

Remark 1.6 We actually prove more. We construct in Section 6 a multiplicative spectral sequence with $E^{2}$ term the algebra $H_{*}\left(\Omega^{2} \mathbb{P}^{n}\right) \otimes H^{*}\left(\mathbb{P}^{n}\right)$ and we show that the only differentials are those obtained via derivations from $d$ above. In other words $\mathbb{H}_{*}\left(L^{2} \mathbb{P}^{n}\right)$ as an algebra has a filtration whose associated graded is isomorphic to the homology described in Theorem 1.5 (1). To obtain the actual multiplicative structure on $\mathbb{H}_{*}\left(L^{2} \mathbb{P}^{n}\right)$ one needs to solve the extension problem at the $E_{\infty}-$ level. To that end it would be useful to work at the chain level as in Félix, Menichi and Thomas [9]. Analogous statements can be made for $\operatorname{Hol}(n)$ in Theorem 1.5 (2). We conjecture that there are no extension problems and that the homology descriptions in (1) and (2) describe respectively $\mathbb{H}_{*}\left(L^{2} \mathbb{P}^{n}\right)$ and $\mathbb{H}_{*}(\operatorname{Hol}(n))$ as algebras.

Corollary 1.7 Let $p$ be a prime.

(1) Collapse If $p$ divides $(n+1)$, then $H_{*}\left(L^{2} \mathbb{P}^{n} ; \mathbb{Z}_{p}\right) \cong H_{*}\left(\Omega^{2} \mathbb{P}^{n} ; \mathbb{Z}_{p}\right) \otimes$ $H_{*}\left(\mathbb{P}^{n} ; \mathbb{Z}_{p}\right)$ and $H_{*}\left(\operatorname{Hol}(n) ; \mathbb{Z}_{p}\right) \cong H_{*}\left(\operatorname{Rat}(n) ; \mathbb{Z}_{p}\right) \otimes H_{*}\left(\mathbb{P}^{n} ; \mathbb{Z}_{p}\right)$.

(2) PERIODICITY Multiplication by $\iota^{p}$ induces a mod-p isomorphism

$$
\mathbb{H}_{*}\left(L_{k}^{2}\left(\mathbb{P}^{n}\right) ; \mathbb{Z}_{p}\right) \cong \mathbb{H}_{*}\left(L_{k+p}^{2}\left(\mathbb{P}^{n}\right) ; \mathbb{Z}_{p}\right)
$$

If $p$ does not divide $k$, then postcomposition by a map $\mathbb{P}^{n} \rightarrow \mathbb{P}^{n}$ of algebraic degree $k$ induces an isomorphism $\mathbb{H}_{*}\left(L_{1}^{2} \mathbb{P}^{n} ; \mathbb{Z}_{p}\right) \cong \mathbb{H}_{*}\left(L_{k}^{2} \mathbb{P}^{n} ; \mathbb{Z}_{p}\right)$.

(3) If $n$ is even, then the spectral sequence computing the homology mod 2 of any component of $L^{2} \mathbb{P}^{n}$ or any positive component of $\operatorname{Hol}(n)$ does not collapse. The same is true if $p$ is odd and $p$ does not divide $k(n+1)$. If $p$ is odd and divides $k$, then the spectral sequences of $L_{k}^{2} \mathbb{P}^{n}$ and $\operatorname{Hol}_{k}(n) \bmod p$ collapse.

Part (3) of this corollary recovers in the case $n=1$ the main result of Havlicek [12].

We finally turn to maps from higher genus curves into $\mathbb{P}^{n}$. In general the homology groups of $\operatorname{Map}\left(C, \mathbb{P}^{n}\right)$ and of its holomorphic subspace $\operatorname{Hol}\left(C, \mathbb{P}^{n}\right)$ are hard to compute and little is known about them [15]. An important homological approximation 
theorem due to Segal [20] states that the inclusion of $k$-th components

$$
i_{k}: \operatorname{Hol}_{k}\left(C, \mathbb{P}^{n}\right) \hookrightarrow \operatorname{Map}_{k}\left(C, \mathbb{P}^{n}\right)
$$

is a homology isomorphism up to dimension $(k-2 g)(2 n-1)$, where $g$ is the genus of $C$. In Section 6 we combine the calculations of Corollary 1.7 to the module structure of Corollary 1.2 to gain the following important insight.

Theorem 1.8 Let $C$ be a compact Riemann surface.

(1) PERIODICITY If $p$ divides $k(n+1)$, then

$$
\mathbb{H}_{*}\left(\operatorname{Map}_{i}\left(C, \mathbb{P}^{n}\right) ; \mathbb{Z}_{p}\right) \cong \mathbb{H}_{*}\left(\operatorname{Map}_{i+k}\left(C, \mathbb{P}^{n}\right) ; \mathbb{Z}_{p}\right)
$$

In particular if $p$ divides $n+1$ then all components have the same homology.

(2) Dichотомy The additive homology of a component of $\operatorname{Map}\left(C, \mathbb{P}^{n}\right)$ with coefficients in a field is isomorphic to the homology of the component of degree either zero or one.

In order to obtain the homology groups component by component, it is necessary to find a model for these mapping spaces that allows for a much deeper insight into their geometry. Such a model built out of configuration spaces is constructed and studied in our sequel [16]. One finds in particular homology torsion of order $k(n+1)$ and $n(n+1)$.

Acknowledgment We wish to thank Daniel Tanré for his constant support. We also thank the referees for improving with their comments earlier versions of this paper.

\section{The degree one component and rational homology}

As will soon be apparent, the geometry of the space of holomorphic maps $\mathbb{P}^{1} \longrightarrow \mathbb{P}^{n}$ is essentially reflected in the degree one component $\operatorname{Hol}_{1}(n)$. We fix base points so that

(2-1) $\operatorname{Rat}(n)=\left\{f: \mathbb{P}^{1} \longrightarrow \mathbb{P}^{n} \mid f\right.$ holomorphic, and $\left.f([1: 0])=[1: 0: \ldots: 0]\right\}$. 
Lemma 2.1 Let $\tau\left(\mathbb{P}^{n}\right)$ be the sphere bundle of unit tangent vectors of $\mathbb{P}^{n}$. Then there is a fiberwise homotopy equivalence:

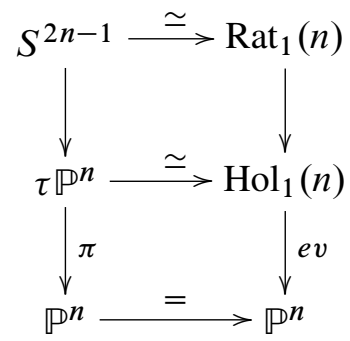

Proof A linear map $\mathbb{P}^{1} \rightarrow \mathbb{P}^{n}$ sending the base point $[1: 0]$ to $[1: 0: \cdots: 0]$ has the form $[z: x] \mapsto\left[z+z_{0} x: z_{1} x: \cdots: z_{n} x\right]$, with $z_{0} \in \mathbb{C}$ and $\left(z_{1}, \ldots, z_{n}\right) \neq 0$, so that $\operatorname{Rat}_{1}(n) \cong \mathbb{C} \times\left(\mathbb{C}^{n}-0\right)$. Since $\mathbb{P}^{n}$ is acted on transitively by $P U(n+1)$, and the stabilizer of the base point is $1 \times U(n)$, there is a diffeomorphism

$$
\operatorname{Hol}_{k}(n) \cong P U(n+1) \times_{U(n)} \operatorname{Rat}_{k}(n)
$$

and a smooth bundle $\operatorname{Rat}_{k}(n) \rightarrow \operatorname{Hol}_{k}(n) \rightarrow \mathbb{P}^{n}$, where $U(n)$ acts on $\operatorname{Rat}_{k}(n)$ by postcomposition. The unit sphere $S^{2 n-1} \subset\{0\} \times\left(\mathbb{C}^{n}-0\right) \subset$ Rat $_{1}(n)$ is a $U(n)-$ invariant subspace and a deformation retract. We identify the tangent space to $\mathbb{P}^{n}$ in $[1: 0: \cdots: 0]$ with the copy $\mathbb{C}^{n} \subset \mathbb{P}^{n}$ embedded via $\left(x_{1}, \ldots, x_{n}\right) \rightarrow\left[1: x_{1}: \cdots: x_{n}\right]$, compatibly with the action of $U(n)$. Then $P U(n+1) \times_{U(n)} S^{2 n-1}$ is the bundle $\tau\left(\mathbb{P}^{n}\right)$ of unit tangent vectors with respect to the Fubini-Study metric. The induced map of bundles $\tau\left(\mathbb{P}^{n}\right) \longrightarrow \operatorname{Hol}_{1}(n)$ is a homotopy equivalence, by comparing the long exact sequences of homotopy groups.

Here is an example of a calculation that does not require Chas-Sullivan products yet. We denote again by $\iota \in H_{0}\left(\operatorname{Rat}_{1}(n) ; \mathbb{Z}\right)$ and $u \in H_{2 n-1}\left(\operatorname{Rat}_{1}(n) ; \mathbb{Z}\right)$ the generators. As discussed in the introduction we have a product

$$
H_{i_{1}}\left(\operatorname{Rat}_{k_{1}}(n) ; \mathbb{Z}\right) \otimes \cdots \otimes H_{i_{r}}\left(\operatorname{Rat}_{k_{r}}(n) ; \mathbb{Z}\right) \longrightarrow H_{i_{1}+\cdots+i_{r}}\left(\operatorname{Rat}_{k_{1}+\cdots+k_{r}}(n) ; \mathbb{Z}\right)
$$

and the class $u \iota^{k-1} \in H_{2 n-1}\left(\operatorname{Rat}_{k}(n) ; \mathbb{Z}\right) \cong \mathbb{Z}$ is a generator [4]

Proposition 2.2 Let $a=\left[\mathbb{P}^{n}\right] \in H_{2 n}\left(\mathbb{P}^{n} ; \mathbb{Z}\right)$ be the fundamental class. Then in the integral homology Serre spectral sequence for $\operatorname{Rat}_{k}(n) \rightarrow \operatorname{Hol}_{k}(n) \rightarrow \mathbb{P}^{n}, d_{2 n}(a)=$ $(n+1) k u \iota^{k-1}$.

Proof In the case $k=0$ we have that $d_{2 n}(a)=0$, since the fiber is a point. The case $k=1$ follows from Lemma 2.1, since in the homology Serre spectral sequence 
for $\tau \mathbb{P}^{n}, d_{2 n}(a)=(n+1) u$, as $(n+1)$ is the Euler characteristic of $\mathbb{P}^{n}$. Now observe that precomposition with the $k$ degree map $\mathbb{P}^{1} \stackrel{\times k}{\longrightarrow} \mathbb{P}^{1}$ given by sending $\left[z_{0}: z_{1}\right] \mapsto\left[z_{0}^{k}: z_{1}^{k}\right]$ takes degree one maps to degree $k$ maps and commutes with the evaluation maps as in the diagram:

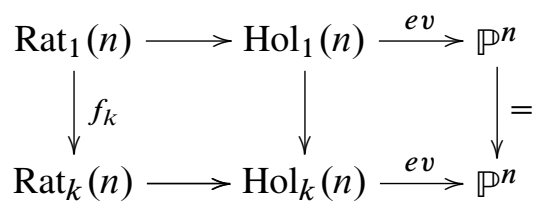

We therefore obtain a map of Serre spectral sequences between the evaluation fibrations, and to prove our claim it suffices by comparison of spectral sequences to show that the fiber map $f_{k}$ induces multiplication by $k$ on $H_{2 n-1} \cong \mathbb{Z}$. But notice that $f_{k}$ extends to the $k$-th power map of the $\mathrm{H}$-space $\Omega^{2} \mathbb{P}^{n}$, the inclusion $H_{*}(\operatorname{Rat}(n)) \rightarrow H_{*}\left(\Omega^{2} \mathbb{P}^{n}\right)$ is a ring monomorphism, and the diagonal induces $\Delta_{*}(u)=u \otimes \iota+\iota \otimes u$. It follows that $f_{k *}(u)=k u \iota^{k-1}$ as desired.

Corollary 2.3 For $k>0, H_{*}\left(\operatorname{Hol}_{k}(n) ; \mathbb{Q}\right) \cong H_{*}\left(\mathbb{P}^{n-1} ; \mathbb{Q}\right) \oplus s^{2 n-1} \widetilde{H}_{*}\left(\mathbb{P}^{n} ; \mathbb{Q}\right)$, where $s^{2 n-1}$ is the formal operator that raises degrees by $2 n-1$.

Proof For $k>0$ there is a rational equivalence $H_{*}\left(\operatorname{Rat}_{k}(n) ; \mathbb{Q}\right) \cong H_{*}\left(\Omega_{k}^{2} \mathbb{P}^{n} ; \mathbb{Q}\right)$ [4]. But $\Omega_{k}^{2} \mathbb{P}^{n} \simeq \Omega^{2} S^{2 n+1}$ is rationally the sphere $S^{2 n-1}$, and its fundamental class is hit by $\left[\mathbb{P}^{n}\right]$ according to Proposition 2.2.

\section{Intersection products and module structures}

In this section we adapt the intersection product of Chas-Sullivan-Cohen-Jones to obtain homology pairings and module maps in slightly more general settings. Our homology pairings are not induced from maps of spaces but are a formal consequence of constructions performed at the level of spectra as first indicated in [6]. A chain level analog of the homology constructions of this section are given in Section 6.

Throughout $M$ will refer to a closed compact $d$-manifold, and $x_{0}$ is a basepoint in $S^{n}$. Let $M \longrightarrow M \times M$ be the diagonal with normal bundle isomorphic to $T M$. The Pontrjagin-Thom collapse map takes $M \times M$ into the Thom space $M^{T M}$. If $\tau$ is any other bundle over $M, M^{\tau}$ its Thom complex, then one can twist the Thom-Pontrjagin construction by $\tau$ and obtain a map of based spaces

$$
M^{\tau} \wedge M^{\tau} \longrightarrow M^{T M \oplus 2 \tau} .
$$


If $\tau$ is a virtual bundle, then the above construction still makes sense at the level of spectra [7]. More precisely, if $M \hookrightarrow \mathbb{R}^{N}$ is an embedding with normal bundle $v$, then the Thom spectrum $M^{-T M}$ is by definition the $N$-fold desuspension of the suspension spectrum of the Thom space $M^{v}$. So set $\tau=-T M$ the virtual normal bundle of $M$. The pairing in (3-1) becomes

$$
M^{-T M} \wedge M^{-T M} \longrightarrow M^{-T M}
$$

and this endows $M^{-T M}$ with the structure of a ring spectrum [5]. It is well known that $M^{-T M}$ is Spanier-Whitehead dual to $M_{+}$, and the multiplication (3-2) is dual to the diagonal $M \rightarrow M \times M$.

Consider the one-point union $X \vee S$ and write $e v$ : Map $(\diamond, M) \longrightarrow M$ the evaluation at the basepoint for $\diamond=M$ or $\diamond=S$. We have a commutative diagram

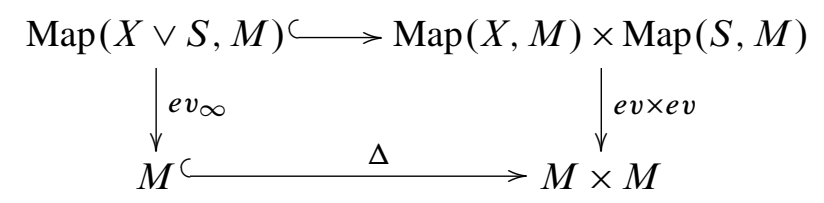

where $e v_{\infty}: \operatorname{Map}(X \vee S, M) \longrightarrow M$ is evaluation at the wedgepoint and the righthand square is a pullback square. The hooked arrows are embeddings of finite codimension and the top such map is a map between infinite dimensional manifolds. We wish to compare the tubular neighborhoods of top and bottom embeddings. The following lemma is used in a special case in [7] but not explicitly stated nor proved there.

Lemma 3.1 Let $X, S$ be based spaces and $M$ a closed compact smooth manifold. Then the continuous mapping space $\operatorname{Map}(X \vee S, M)$ has a neighbourhood in $\operatorname{Map}(X, M) \times \operatorname{Map}(S, M)$ homeomorphic to the pullback of the tangent bundle of $M$ along the evaluation map at the wedgepoint.

Proof The point is to construct a tubular neighbourhood of the top right embedding in diagram (3-3) of the form $(e v \times e v)^{-1}(U)$ where $U$ is a tubular neighborhood of the diagonal $\Delta: M \longrightarrow M \times M$ and with the main property that if $(D M, S M)$ is the pair (disc,sphere)-bundles associated to the tangent bundle of $M$, then up to homeomorphism

$$
\left((e v \times e v)^{-1}(U),(e v \times e v)^{-1}(\partial U)\right) \cong\left(e v_{\infty}^{*} D M, e v_{\infty}^{*} S M\right)
$$

and these in turn are identified with $\left(\widetilde{v}_{D}, \widetilde{v}_{S}\right)$, the pair (disc,sphere)-bundles associated to the pullback bundle $\tilde{v}=e v_{\infty}^{*}(T M)$.

Choose a metric on $M$ with injectivity radius greater than 2 and let as above $D M \subset T M$ be the unit disc bundle of the tangent bundle of $M$. We will write exp the exponential 
map $D M \longrightarrow M$ and $\exp _{x}$ its restriction to $D_{x} M:=T_{x} M \cap D M$, for $x \in M$. The image $U \subset M \times M$ of $D M$ via $(x, v) \mapsto\left(\exp _{x}(v)\right.$, $\left.\exp _{x}(-v)\right)$ is a tubular neighbourhood of the submanifold $\operatorname{Im}(\Delta) \subset M \times M$.

We now build a map EXP: $D M \rightarrow \operatorname{Homeo}(M)$ to the group of self homeomorphisms of $M$ such that, for $(x, v) \in D_{x}(M), \operatorname{EXP}(x, v)=\exp _{x}(v)$.

For $(x, v) \in D_{x} M$, consider the self map $\phi_{v}: T_{x}(M) \rightarrow T_{x}(M)$ that is the identity on vectors of norm $\geq 2$ and such that $\phi_{v}(w)=w+(1-|w| / 2) v$ for $w \in T_{x}(M)$ and $|w| \leq 2$. This map is a homeomorphism taking the origin to $v$. The composition $\exp _{x} \circ \phi_{v} \circ \exp _{x}^{-1}$ extended by the identity outside of the exponential image of the radius 2 disc at $x$ defines the self homeomorphism $\operatorname{EXP}(x, v)$ of $M$.

With $e v_{\infty}$ as in (3-3), the pullback $e v_{\infty}^{*}(D M)$ consists of pairs $\left(\left(f_{1}, f_{2}\right),(p, v)\right)$ where $e v\left(f_{1}\right)=e v\left(f_{2}\right)=p$ and $v \in T_{p} M,|v| \leq 1$. Consider then the map

$$
\phi: e v_{\infty}^{*}(D M) \longrightarrow \operatorname{Map}(X, M) \times \operatorname{Map}(S, M)
$$

sending $\left(\left(f_{1}, f_{2}\right),(p, v)\right) \longmapsto\left(\operatorname{EXP}(p, v) \circ f_{1}, \operatorname{EXP}(p,-v) \circ f_{2}\right)$. This map is oneto-one because the injectivity radius is greater than 2 . The map $\phi$ is also an embedding onto $(e v \times e v)^{-1}(U)$. Indeed, for base points $x_{0} \in X$ and $s_{0} \in S$, suppose that $g: X \rightarrow M$ and $h: S \rightarrow M$ are such that $\left(g\left(x_{0}\right), h\left(s_{0}\right)\right) \in U$. Then there exist $x \in M$ and $v \in T_{x}(M)$ such that $g\left(x_{0}\right)=\exp _{x}(v)$ and $h\left(s_{0}\right)=\exp _{x}(-v)$. Let us set $f_{1}=\operatorname{EXP}(x, v)^{-1} \circ g$ and $f_{2}=\operatorname{EXP}(x,-v)^{-1} \circ h$. By construction $\operatorname{EXP}(x, v)(x)=\exp _{x}(v)=g\left(x_{0}\right)$, so that $f_{1}\left(x_{0}\right)=x$. Similarly $f_{2}\left(s_{0}\right)=x$. Then $(g, h)$ is $\phi\left(\left(f_{1}, f_{2}\right),(x, v)\right)$ and $\phi$ is surjective onto $(e v \times e v)^{-1}(U)$ as claimed.

Remark 3.2 Observe that the existence of the above neighborhood depended essentially on the fact that a smooth (connected) manifold is a "homogeneous" space Homeo $(M) /$ Homeo* $^{*}(M)$ of all homeomorphisms modulo based homeomorphisms. An analog of this construction in the holomorphic category appears in Lemma 5.1.

Going back to (3-3), the Thom-Pontrjagin construction with respect to the pullback $e v_{\infty}^{*} T M$, which we also write $T M$, yields then a map $\operatorname{Map}(X, M) \times \operatorname{Map}(S, M) \longrightarrow$ $\operatorname{Map}(X \vee S, M)^{T M}$ which covers via the evaluations the map $M \times M \longrightarrow M^{T M}$. We can twist as before by $\tau=-T M$ and obtain the diagram of Thom spectra

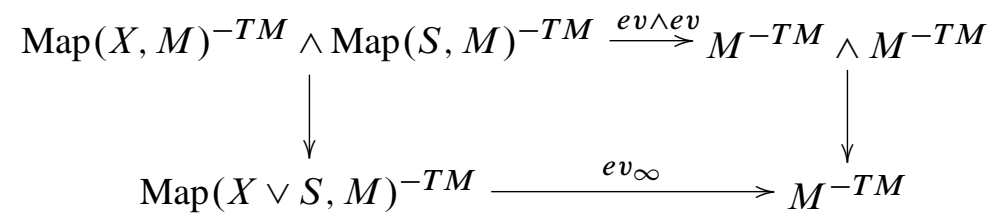


We use this diagram of spectra to derive Theorem 1.1 of the introduction.

Proof of Theorem 1.1 Let $S$ be a co- $H$ space and set $X=S$. Consider the composite

$$
\begin{aligned}
& \mu: \operatorname{Map}(S, M)^{-T M} \wedge \operatorname{Map}(S, M)^{-T M} \longrightarrow \operatorname{Map}(S \vee S, M)^{-T M} \\
& \stackrel{m}{\longrightarrow} \operatorname{Map}(S, M)^{-T M}
\end{aligned}
$$

where the first map is the left vertical of (3-4) and the second map is induced from the co- $H$ structure $S \longrightarrow S \vee S$. We need show that: (i) $\mu$ has a unit, (ii) is commutative if $S$ is cocommutative and (iii) is associative if $S$ is coassociative. As pointed out in [7], the unit is the composite $S^{0} \longrightarrow M^{-T M} \longrightarrow \operatorname{Map}(S, M)^{-T M}$ where the last map is induced from the canonical section $M \longrightarrow \operatorname{Map}(S, M)$ sending $x \in M$ to the constant map at $x$.

Let's simplify the notation by setting $P:=\operatorname{Map}(S, M), P \times_{M} \cdots \times_{M} P:=\operatorname{Map}(S \vee$ $\cdots \vee S, M)$. To show (ii) notice there is an involution $\tau$ on $P \times_{M} P=\operatorname{Map}(S \vee S, M)$ which comes from permuting both factors in $S \vee S$. When $S$ is cocommutative, $m \circ \tau \simeq m$ so that the composite at the level of spectra

$$
P^{-T M} \wedge P^{-T M} \longrightarrow\left(P \times_{M} P\right)^{-T M} \stackrel{\tau^{-T M}}{\longrightarrow}\left(P \times_{M} P\right)^{-T M} \longrightarrow P^{-T M}
$$

is homotopic to $\mu$. Associativity (iii) on the other hand follows from the diagram:

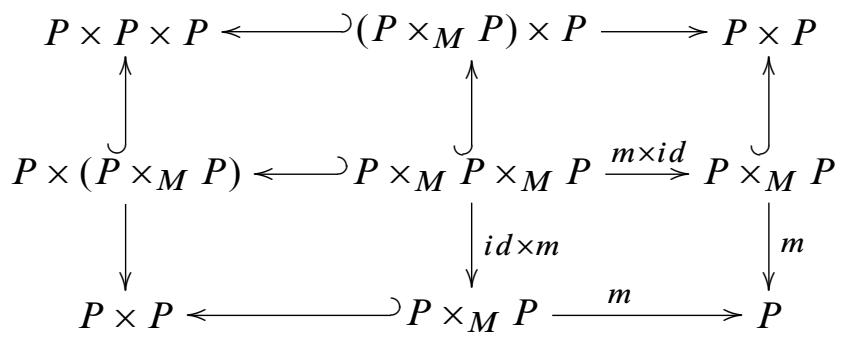

The lower-right square commutes because $S$ is coassociative. The other three subsquares are pullbacks, and the hooked arrows are embeddings with normal bundle isomorphic to the pullback of $T M$. Then we can apply the Thom-Pontrjagin construction twisted by $-T M$. This changes the direction of the hooked arrows in diagram (3-5) and produces a new diagram (3-5)' at the level of Thom spectra. The top left diagram of (3-5)' homotopy commutes just because (a) it is obtained by taking the Thom-Pontrjagin construction with respect to two tubular neighborhoods of $P \times_{M} P \times_{M} P \hookrightarrow P \times P \times P$ obtained as pullbacks of tubular neighborhoods of the thin diagonal in $M^{3}$, and (b) the Thom-Pontrjagin construction doesn't depend up 
to homotopy on the choice of tubular neighborhood. The three subsquares of (3-5)' coming from pullback squares are hence homotopy commutative and thus the outer square of that diagram is homotopy commutative which proves associativity.

The proof for the module structure is completely analogous. In that case the analog of $\mu$ is obtained precomposing by the coaction map $X \rightarrow X \vee S$ and the analog of (3-5) is obtained by replacing the rightmost $P$ 's by $\operatorname{Map}(X, M)$ throughout.

Remark 3.3 The arguments above show more generally that if $X, Y$ and $Z$ are spaces then there is a map

$$
\begin{aligned}
\operatorname{Map}(X, M)^{-T M} \wedge \operatorname{Map}(Y, M)^{-T M} & \wedge \operatorname{Map}(Z, M)^{-T M} \\
& \longrightarrow \operatorname{Map}(X \vee Y \vee Z, M)^{-T M}
\end{aligned}
$$

which is well-defined up to homotopy (ie, the appropriate associativity diagram commutes up to homotopy).

\subsection{Homology version}

We now pass from ring and module spectra to homology pairings. As in [6], let $h_{*}$ be any generalized homology theory such that (i) the associated cohomology theory $h^{*}$ is multiplicative, that is the underlying spectrum is a ring spectrum, and (ii) $M$ is $h_{*}$-oriented to ensure the existence of the Thom isomorphism [21, Chapter 13]. Let $\wedge$ be the intersection pairing on the homology $h_{*}(M)=\bigoplus_{0 \leq i \leq d} h_{i}(M)$ induced from the Thom isomorphism with respect to the diagonal embedding. This relates to the cohomology product in $h^{*}$ via generalized Poincaré duality $p d$ according to the diagram:

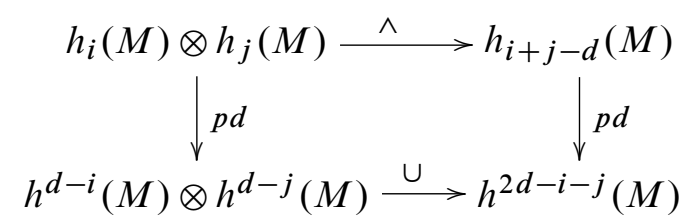

The following is a direct consequence of the commutativity of diagram (3-3).

Corollary 3.4 Let $M$ be an $h_{*}$-oriented manifold of dimension $d, S$ a co- $H$ space coacting on $X$. There is a commutative diagram:

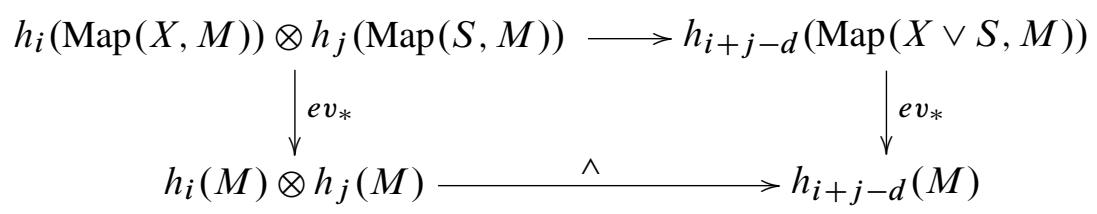


This gives a pairing $\bullet$ making $h_{d+*}(\operatorname{Map}(S, M))$ into a graded ring which is commutative if $S$ is cocommutative, and $h_{d+*}(\operatorname{Map}(N, M))$ into a graded module over $h_{d+*}(\operatorname{Map}(S, M))$.

Proof Let $m: X \rightarrow X \vee S$ be the coaction map. To obtain the pairing $\bullet$ apply $h_{*}$ to diagram (3-3) and use the Thom-Pontrjagin construction to get an Umkehr map going the "wrong way" with the appropriate shift by codimension of the hooked arrow (the embedding). This is depicted as follows

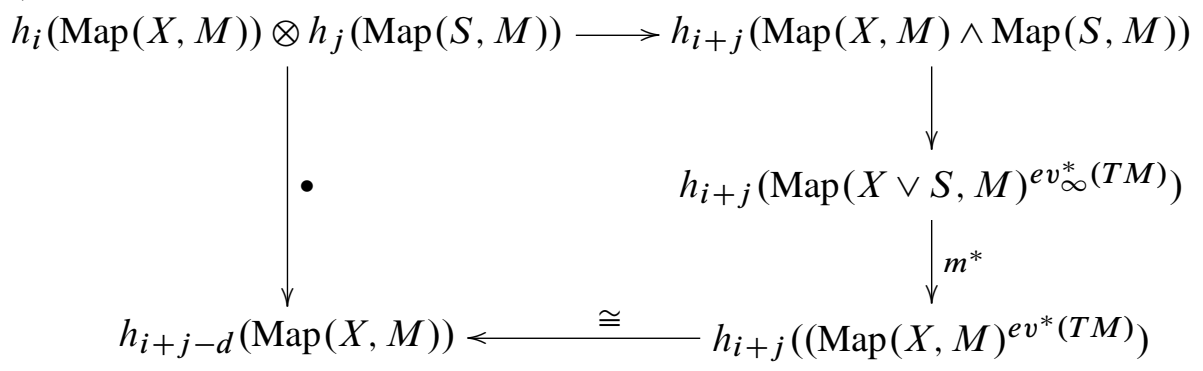

where the top map is the slant pairing $[21,13.70]$ and the bottom map is the Thom isomorphism which is valid since $M$ oriented [21, Theorem 14.6]. The composite in (3-8) is the desired pairing - compatible via the evaluation morphisms and according to Lemma 3.1 to the intersection pairing on $h_{*}(M)$ as in (3-7).

When $S$ is a co-associative co- $H$ space, the associativity of $\bullet$ is a direct consequence of diagram (3-5). Indeed consider the outer square in homology (again homology is contravariant on all hooked arrows). Starting with $x \otimes y \otimes z \in h_{*}(P \times P \times P)$ and going clockwise we obtain $x \bullet(y \bullet z) \in h_{*-2 d}(P)$. Going counterclockwise we obtain $(x \bullet y) \bullet z$. A similar diagram gives the module structure.

Proof of Corollary 1.2 The first part follows by setting $S=S^{n}$. In this case $\mathbb{H}_{*}\left(L^{n} M\right):=H_{*+d}\left(L^{n} M\right)$ is a graded commutative ring. The commutativity follows for $n>1$ from the fact that $S^{n}$ is cocommutative and when $n=1$ from the action of the circle on the loop space by rotations [2]. We observe that this ring has been constructed for $n=1$ in [7] and for $n>1$ in [13] and in [3] using geometric bordism theory.

For the second part, we observe that a closed based manifold $N$ of dimension $n$ is the cofiber of the top cell attaching map. The associated coaction map $N \longrightarrow N \vee S^{n}$ is obtained geometrically by pinching the boundary of a small closed disc neighborhood of the basepoint in $N$. 
We will refer to the module structure map

$$
\bullet: \mathbb{H}_{*}\left(L^{n} M\right) \otimes \mathbb{H}_{*}(\operatorname{Map}(N, M)) \longrightarrow \mathbb{H}_{*}(\operatorname{Map}(N, M))
$$

as "intersection module" map from now on.

\section{Holomorphic products I}

Let us specialize the diagram (3-3) to the case $N=S^{2}$

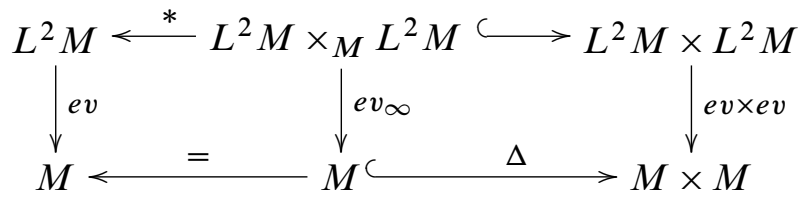

In this section and the next we propose to exhibit a commutative diagram as in (4-1) with $L^{2} M$ replaced by $\operatorname{Hol}(M)$ where $\operatorname{Hol}(M)$ indicates the space of holomorphic maps from $\mathbb{P}^{1}$ to a complex projective homogeneous $n$-manifold $M$. As a direct consequence we obtain a "holomorphic" intersection product

$$
H_{i}(\operatorname{Hol}(M)) \otimes H_{j}(\operatorname{Hol}(M)) \longrightarrow H_{i+j-2 n}(\operatorname{Hol}(M))
$$

We give first the construction for $M=\mathbb{P}^{n}$ since it is both simpler and more explicit than the more general case. The point here is to exploit the fact that the $\mathrm{H}$-space structure of the based holomorphic maps Rat $(n)$ can be defined by adding vectors of rational functions. In general this addition is neither well-defined nor continuous on the entire space, but its definition makes sense on a deformation retract of $\operatorname{Rat}(n) \times \operatorname{Rat}(n)$.

Proof of Theorem 1.3 for $G / P=\mathbb{P}^{n} \quad$ Write $\mathbb{P}^{1}=\mathbb{C} \cup\{\infty\}$ and let $\operatorname{Rat}(n)$ be as in (2-1). If $f \in \operatorname{Rat}_{k}(n)$, then the restriction of $f$ to $\mathbb{C}$ is of the form $f(z)=[g(z)$ : $\left.p_{1}(z): \cdots: p_{n}(z)\right]$, the components being polynomials with $\operatorname{deg} p_{i}<\operatorname{deg} g=k$, and such that $g, p_{1}, \ldots, p_{n}$ have no common zero. We therefore identify $f$ with its corresponding vector of rational functions $\left(p_{1} / g, \ldots, p_{n} / g\right)$. We call the zeroes of $g$ the poles of $f$.

Now $P U(n+1)$ acts on $\mathbb{P}^{n}$ by isometries and the stabilizer of the point $[1: 0$ : ․: 0$]$ is a copy of $U(n) \subset P U(n+1)$. It follows that $U(n)$ acts on $\operatorname{Rat}(n)$ by postcomposition and the action is given by matrix multiplication of $A \in U(n)$ and a vector $f=\left(p_{1} / g, \ldots, p_{n} / g\right) \in \operatorname{Rat}(n)$. We remark that $A f$ and $f$ have the same poles.

Let $\operatorname{Rat}_{\leq i}(n)$ be the $U(n)$-invariant subspace of $\operatorname{Rat}(n)$ consisting of maps $f$ with poles of norm less than $i$. The inclusion $\operatorname{Rat}_{\leq i}(n) \subset \operatorname{Rat}_{\leq 2 i}(n)$ has a $U(n)$-equivariant 
homotopy inverse sending $f(z)$ to $f(2 z)$, since $U(n)$ acts by postcomposition, and the action commutes with precomposition. This induces in turn a homotopy equivalence

$$
P U(n+1) \times_{U(n)} \operatorname{Rat}_{\leq i}(n) \stackrel{\simeq}{\longrightarrow} P U(n+1) \times_{U(n)} \operatorname{Rat}_{\leq 2 i}(n)
$$

and in the limit we obtain a homotopy equivalence

$$
P U(n+1) \times_{U(n)} \operatorname{Rat}_{\leq 1}(n) \simeq P U(n+1) \times_{U(n)} \operatorname{Rat}(n)=\operatorname{Hol}(n) .
$$

The same argument as above when applied to translations $f(z) \mapsto f(z-c)$ shows that $P U(n+1) \times_{U(n)} \operatorname{Rat}^{u}(n)$ and $P U(n+1) \times_{U(n)} \operatorname{Rat}^{l}(n)$ are homotopy equivalent to $\operatorname{Hol}(n)$, where $\mathrm{Rat}^{u}(n)$ and $\mathrm{Rat}^{l}(n)$ consist of functions having poles in some unit disc in the upper and lower half-planes respectively. Here again $\operatorname{Rat}^{u}(n)$ and $\operatorname{Rat}^{l}(n)$ are $U(n)$-invariant subspaces since the action by $U(n)$ on a given rational function fixes the poles. Similarly, we have a homotopy equivalence $P U(n+1) \times_{U(n)}\left(\operatorname{Rat}^{u}(n) \times\right.$ $\left.\operatorname{Rat}^{l}(n)\right) \simeq P U(n+1) \times_{U(n)}(\operatorname{Rat}(n) \times \operatorname{Rat}(n)) \cong \operatorname{Hol}(n) \times_{\mathbb{p} n} \operatorname{Hol}(n)$. Here $U(n)$ is acting diagonally, and $\operatorname{Hol}(n) \times \mathbb{p} n \operatorname{Hol}(n)$ is the space of pairs of holomorphic maps which agree at the base point.

There is then a map

(4-2) $\operatorname{Hol}_{i}(n) \times \mathbb{P} n \operatorname{Hol}_{j}(n) \simeq P U(n+1) \times U(n)\left(\operatorname{Rat}_{i}^{u}(n) \times \operatorname{Rat}_{j}^{l}(n)\right) \stackrel{+}{\longrightarrow} \operatorname{Hol}_{i+j}(n)$.

Here + is induced by the sum of vectors of rational functions $\operatorname{Rat}_{i}^{u}(n) \times \operatorname{Rat}_{j}^{l}(n) \longrightarrow$ $\operatorname{Rat}_{i+j}(n)$. Such operation is now well defined, since we sum functions with distinct poles, and it is $U(n)$-equivariant, since $A(f+g)=A f+A g$.

The twisted Thom-Pontrjagin construction with respect to the pullback of the tangent bundle $T \mathbb{P}^{n}$, seen as a normal bundle of the inclusion $\operatorname{Hol}_{i}(n) \times \mathbb{p} n \operatorname{Hol}_{j}(n) \hookrightarrow \operatorname{Hol}_{i}(n) \times$ $\mathrm{Hol}_{j}(n)$ (see Lemma 5.1), followed by composition pairing yields the ring spectrum product

$$
\operatorname{Hol}_{i}(n)^{-T \mathbb{P}^{n}} \wedge \operatorname{Hol}_{j}(n)^{-T \mathbb{P}^{n}} \rightarrow\left(\operatorname{Hol}_{i}(n) \times \mathbb{p} n \operatorname{Hol}_{j}(n)\right)^{-T \mathbb{p}^{n}} \rightarrow \operatorname{Hol}_{i+j}(n)^{-T \mathbb{P}^{n}}
$$

The product is homotopy commutative because $\operatorname{Rat}(n)$ is homotopy commutative by a $U(n)$-equivariant homotopy.

Proof of Proposition 1.4 Consider the right-half of diagram (4-1) (the pullback diagram). There is a holomorphic version of it mapping to (4-1) via inclusions. The Thom spaces of the corresponding normal bundles map to each other and hence we get 
a homotopy commutative diagram of spectra

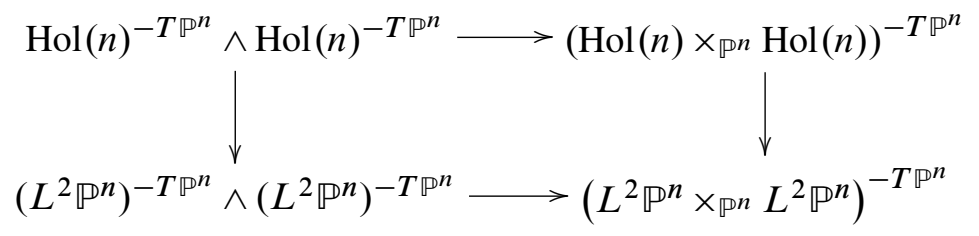

Composing respectively by (4-2) and the loop sum to the right we end up respectively in $\operatorname{Hol}(n)^{-T \mathbb{P}^{n}}$ and $\left(L^{2} \mathbb{P}^{n}\right)^{-T \mathbb{P}^{n}}$. We need show these compositions are compatible and to that end it suffices to show that the following diagram commutes up to $U(n)-$ equivariant homotopy

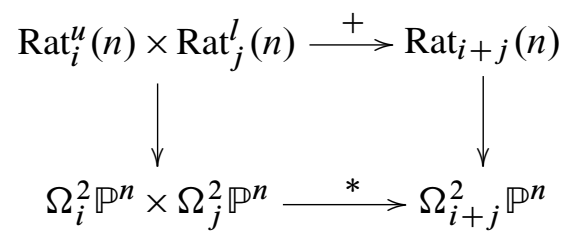

The homotopy is given by $H_{t}(f, g)=f a_{t}+g b_{t}$, where $a_{t}$ (respectively $\left.b_{t}\right)$ is a homotopy between the identity of $S^{2}=\mathbb{C} \cup\{\infty\}$ and the composite $S^{2} \rightarrow S^{2} \vee S^{2} \rightarrow S^{2}$ of the pinch map and the projection onto the first (respectively second) summand. Here $a_{0}=b_{0}=i d$, and $a_{1}: \mathbb{C} \cup\{\infty\} \longrightarrow \mathbb{C} \cup\{\infty\}$ (resp. $b_{1}$ ) sends the lower half-plane (resp. upper half-plane) to $\infty$. Note that $f a_{t}+g b_{t}$ is not holomorphic anymore for $t>0$, however the deformations $a$ and $b$ can be chosen so that the "poles" of $f a_{t}$ (ie, the zeros of $p_{0, t}$ if we write $f a_{t}=\left[p_{0, t}: p_{1, t}: \ldots: p_{n, t}\right]$ ) and those of $g b_{t}$ remain fixed as $t$ varies. This ensures that $f a_{t}+g b_{t}$ is still well defined as a map into $\mathbb{P}^{n}$.

Remark 4.1 It is more common in the literature (eg [4]) to define instead

$$
\operatorname{Rat}(n)=\left\{f: \mathbb{P}^{1} \longrightarrow \mathbb{P}^{n} \mid f \text { holomorphic, and } f([1: 0])=[1: 1: \ldots: 1]\right\} .
$$

In this case a function $f \in \operatorname{Rat}(n)$ is identified with a vector $\left(f_{1} / g, \ldots, f_{n} / g\right)$, such that $\operatorname{deg} f_{i}=\operatorname{deg} g=k$ and $f_{1}, \ldots, f_{n}, g$ are monic polynomials without common zeroes. In this case the componentwise product of rational functions on a deformation retract of $\operatorname{Rat}(n) \times \operatorname{Rat}(n)$ defines an $\mathrm{H}$-space structure, similarly as before. It turns out that the product and the sum give two $\mathrm{H}$-space structures on Rat $(n)$ which are equivalent. More generally there are $C_{2}$-structures associated to these operations that are equivalent as well. However the product is less suitable here since it comes from the description of $\mathbb{P}^{n}$ as a toric variety rather than a homogeneous space. 


\section{Holomorphic products II}

We now extend the product on $\operatorname{Hol}\left(\mathbb{P}^{n}\right)$ to $\operatorname{Hol}(M) \subset L^{2} M$ where $M$ is a homogeneous projective variety. This is based on a beautiful extension of the root-pole description of holomorphic maps for the case of projective space to this more general situation as first formulated by Gravesen and Segal, and then greatly exploited in [1].

We follow the notation of [1] and consider a complex connected semisimple Lie group $G$ with Lie algebra $\mathfrak{g}$. Choose a Cartan subalgebra $\mathfrak{h}$ and the associated positive and negative root spaces $\mathfrak{u}^{+}$and $\mathfrak{u}^{-}$. A parabolic subalgebra $\mathfrak{p}$ is obtained by adjoining to $\mathfrak{h}$ all positive and some negative root subspaces. The corresponding subgroup $P$ is also called parabolic, and $M=G / P$ is a generalized flag manifold, the most general form of a complex projective homogeneous manifold. Let us write $\mathbb{H}_{*}(\operatorname{Hol}(G / P))=H_{*+2 d}(\operatorname{Hol}(G / P))$, if $G / P$ has complex dimension $d$.

Proof of Theorem 1.3 The complement $\mathfrak{n}^{-}$of $\mathfrak{p}$ in $\mathfrak{g}$ corresponds to a contractible group $N \subset G$, such that the projection $N \subset G \rightarrow G / P=M$ identifies $N$ to an open set of $M$ (called the affine part of $M$ ), whose complement is the divisor at infinity $M_{\infty}$. Alternatively and under this identification $N$ is the open cell in the Schubert decomposition for $G / P\left[1\right.$, Section 2]. If $M=\mathbb{P}^{n}$ then $N \cong \mathbb{C}^{n}$ and $M_{\infty}$ is the hyperplane at infinity $\mathbb{P}^{n-1}$.

Let us choose as base point $[P] \in G / P$, that corresponds to the unit $e \in N \subset G / P$. A based holomorphic map $f: \mathbb{P}^{1} \rightarrow M, f(\infty)=e$, has by definition poles at the elements of $f^{-1}\left(M_{\infty}\right)$ which form a finite set. The poles of $f$ together with their associated "principal parts" which we next define, determine entirely the space Rat $(G / P)$ of based holomorphic maps, very much as they do classically for meromorphic maps into $\mathbb{P}^{1}$.

Consider the sheaf of meromorphic functions $\mathcal{M}(G / P)$ on $\mathbb{P}^{1}$ defined so that $\mathcal{M}(G / P)(\mathcal{U}), \mathcal{U}$ open in $\mathbb{P}^{1}$, consists of holomorphic functions $\mathcal{U} \rightarrow G / P$ with image not entirely contained in the divisor at infinity $M_{\infty}$. Let $O(N)$ be the sheaf defined on open sets $\mathcal{U}$ of $M$ by $O(N)(\mathcal{U}):=\operatorname{Hol}(\mathcal{U}, N)$ the space of holomorphic maps into $N$. The action of $N$ on $G / P$ defines an action of $\operatorname{Hol}(\mathcal{U}, N)$ on $\operatorname{Hol}(\mathcal{U}, G / P)$ for any open $\mathcal{U}$ and hence a quotient sheaf $\mathcal{M}(G / P) / O(N)$ which is commonly referred to as the sheaf of principal parts $\mathcal{P} \mathcal{P}$. A global section of $\mathcal{P} \mathcal{P}$ is called a configuration of principal parts, and corresponds to a finite number of points $z_{i}$ on $\mathbb{P}^{1}$ (the poles) labeled by elements of their stalk $\mathcal{P} \mathcal{P}_{z_{i}}$ [14]. Notice that the stalk does not depend on its location since one can translate maps from point to point by an automorphism of $\mathbb{P}^{1}$. The natural map from $\operatorname{Rat}(G / P)$ to the configuration space of principal parts without poles at infinity is a homeomorphism by the results in [1]. The illustrative example of $G / P=\mathbb{P}^{n}$ is discussed at the end of this section. 
This description defines on $\operatorname{Rat}(G / P)$ a homotopy associative and commutative $H-$ space structure. The sum is obtained by first defining homotopies which separate the poles of some given two functions into disjoint discs in $\mathbb{C}$ and then taking the union of the new configurations of principal parts (ie the homotopies change the poles but not the elements in the stalks). A similar procedure defines a $C_{2}$-space structure.

We now turn to the unbased mapping space $\operatorname{Hol}(G / P)$. We let $P$ act as before on the right of $G$ and on the left of $G / P$ and hence of $\operatorname{Rat}(G / P)$. The map $G \times \operatorname{Rat}(G / P) \rightarrow$ $\operatorname{Hol}(G / P)$ given by $(g, f(z)) \mapsto g \cdot f(z)$ induces a homeomorphism $G \times{ }_{P} \operatorname{Rat}(G / P) \cong$ $\operatorname{Hol}(G / P)$, because $G$ acts transitively on $G / P$ by biholomorphisms and $P$ is the stabilizer of the base point.

As in (4-2) we wish to produce a map from $(\operatorname{Rat}(G / P) \times \operatorname{Rat}(G / P)) \times{ }_{P} G$ into $\operatorname{Hol}(G / P)$. There is however a problem in that the action of $P$ does not behave well with respect to our principal parts description; in particular $P$ does not act on $N$ (viewed as a subset of $G / P$ ). To circumvent these issues, we proceed as follows.

Let $U \subset P$ be a maximal compact subgroup contained in the reductive part of $P$. More explicitly let $\mathfrak{P}^{-}$be the set of those negative roots $\alpha$ such that $\mathfrak{g}_{\alpha} \subset \mathfrak{p}$. The Levi decomposition [18] gives $\mathfrak{p}=\mathfrak{r} \oplus \mathfrak{n}$, where

$$
\mathfrak{r}=\mathfrak{h} \oplus \bigoplus_{\alpha \in \mathfrak{P}^{-}}\left(\mathfrak{g}_{\alpha} \oplus \mathfrak{g}_{-\alpha}\right),
$$

and

$$
\mathfrak{n}=\bigoplus_{\alpha \notin \mathfrak{P}^{-}, \alpha<0} \mathfrak{g}_{\alpha}
$$

is the radical nilpotent ideal. Let $R$ be the Lie group of $\mathfrak{r}$. This is a deformation retract of $P$. We have that $R$ normalizes $N$ since $\left[\mathfrak{r}, \mathfrak{n}^{-}\right]=\mathfrak{n}^{-}$. Let $U \subset R$ be a maximal compact subgroup. Then $U$ is also maximal in $P$. If $V$ is a maximal compact subgroup of $G$ containing $U$ then there is an identification $V / U=G / P$.

Consider the left action of $U$ on $G / P$. The subspace $N \subset G / P$ is $U$-invariant because $U$ is contained in the normalizer of $N$. Note that the action on $N$ can be identified to the adjoint action. The complement of $N$ in $G / P$, the divisor at infinity, is clearly $U$-invariant and thus the induced $U$-action on $\operatorname{Rat}(G / P)$ does not change the poles. Similarly $U$ acts on the sheaf $\mathcal{M}(G / P)$ by pointwise multiplication on the left. Since the subsheaf $O(N)$ is $U$-invariant, we get an induced action of $U$ on the quotient sheaf $\mathcal{P} \mathcal{P}$. The action on stalks gives in turn an action on configurations of principal parts which corresponds to the action on $\operatorname{Rat}(G / P)$ under the natural identification. It follows that the $\mathrm{H}$-space sum of $\operatorname{Rat}(G / P)$ is $U$-equivariant. 
The construction of the intersection product follows now as in the case of projective spaces, since $\operatorname{Hol}(G / P)=V \times_{U} \operatorname{Rat}(G / P)$. We however need the Thom-Pontrjagin construction and this makes sense if a suitable tubular neighborhood can be constructed for the subspace of composable holomorphic maps. This is provided by the following lemma.

Lemma 5.1 The subspace $\operatorname{Hol}(G / P) \times_{G / P} \operatorname{Hol}(G / P) \subset \operatorname{Hol}(G / P)^{2}$ has a tubular neighbourhood homeomorphic to the pullback of the tangent bundle along the evaluation map.

Proof The total space of the tangent bundle of $G / P=V / U$ is $T(V / U)=V \times_{U} \mathfrak{v} / \mathfrak{u}$, where $U$ acts by the adjoint representation, and $\mathfrak{v}, \mathfrak{u}$ are the Lie algebras associated to $V, U$. We fix an invariant metric on $V$, that induces a metric on $V / U$. Then we can identify $T(V / U)$ to $V \times_{U} \mathfrak{u}^{\perp} \subset V \times_{U} \mathfrak{v}$. The exponential map $\exp _{V}: \mathfrak{v} \rightarrow V$ can then be used to define a map $E: T(V / U) \rightarrow V$ by $E[g, w]=g \exp _{V}(w) g^{-1}$, for $g \in V$ and $w \in \mathfrak{u}^{\perp} \subset \mathfrak{v}$. The value does not depend on the chosen representative. Let $\Phi: V \times V / U \rightarrow V / U$ be the natural action. Then $\Phi(E[g, w], g U)$ agrees with $\exp _{V / U}[g, w]$, where $\exp _{V / U}: T_{g U}(V / U) \rightarrow V / U$ is the exponential map of $V / U$. Since $V$ acts on $V / U$ by biholomorphisms, for each $v \in V$ and holomorphic map $z \mapsto f(z) \in V / U$ the map $z \mapsto \Phi(v, f(z)) \in V / U$ is also holomorphic.

The tubular neighbourhood consists of pairs $\Phi\left(E[g, w], f_{1}(z)\right), \Phi\left(E[g,-w], f_{2}(z)\right)$, where $f_{1}, f_{2}: \mathbb{P}^{1} \rightarrow G / P$ are such that $f_{1}(\infty)=f_{2}(\infty)=g U$ and $[g, w] \in T_{g U}(G / P)$ is shorter than the injectivity radius. This tubular neighbourhood is the preimage via $e v \times e v$ of a tubular neighbourhood for $\Delta: G / P \rightarrow(G / P)^{2}$.

Remark 5.2 For $f \in \operatorname{Rat}(G / P)$, the image curve $f\left(\mathbb{P}^{1}\right)$ intersects each irreducible component of the divisor $M_{\infty}$ into a finite number of points (with multiplicities), and the vector of integers so defined determines the connected components of $\operatorname{Rat}(G / P)$.

\subsection{Example : Complex projective space}

Think of $\mathbb{P}^{n}$ as $S L(n+1, \mathbb{C}) / P$ where $P$ is the subgroup of matrices of the form $\left(\begin{array}{ll}a & B \\ 0 & C\end{array}\right)$ with $C$ of size $n \times n$ and $a \in \mathbb{C}^{*}$. Then $\mathbb{C}^{n}$ is identified with the subgroup of matrices

$$
\left(\begin{array}{ccc}
1 & 0 \cdots 0 \\
z_{1} & 1 \cdots 0 \\
\vdots & \vdots \\
z_{n} & 0 \cdots 1
\end{array}\right)
$$


which acts on $S L(n+1, \mathbb{C}) / P$ by matrix multiplication on the left. Note that this action leaves invariant the hyperplane at infinity which corresponds to those matrices with the top left entry zero. If we choose $U=P \cap S U(n+1)$ and $V=S U(n+1)$, then we have the identification $\mathbb{P}^{n}=S L(n+1, \mathbb{C}) / P=V / U$. Given $f: \mathbb{P}^{1} \longrightarrow S L(n+1) / P$, then away from the poles it has matrix representation

$$
\left(\begin{array}{cc}
1 & 0 \cdots 0 \\
p_{1} / g & 1 \cdots 0 \\
\vdots & \vdots \\
p_{n} / g & 0 \cdots 1
\end{array}\right)
$$

with reference to Section 4.

The action of the sheaf of holomorphic functions $h=\left(h_{1}, \ldots, h_{n}\right)$ (away from the poles) corresponds to pointwise addition of functions with values in $N=\mathbb{C}^{n}$. It is easy then to see that a principal part of a pole at $c \in \mathbb{C}$ is a $n$-tuple of elements of form $\sum_{i=1}^{j} a_{i}(z-c)^{-i}$, seen as an equivalence class of germs of meromorphic functions modulo holomorphic functions. The pointwise addition of meromorphic functions with distinct poles keeps the principal parts of each function. This recovers the $\mathrm{H}$-space structure of Section 4.

Proof of Theorem 1.3 in the general case We mimic the construction for the continuous case and observe that: (i) a diagram as in (4-1) holds if we replace the functor $L^{2}$ by Hol, (ii) The Thom-Pontrjagin construction with respect to the tubular neighborhood constructed in Lemma 5.1 yields a map $\operatorname{Hol}(G / P) \times \operatorname{Hol}(G / P) \longrightarrow\left(\operatorname{Hol}(G / P) \times_{G / P}\right.$ $\operatorname{Hol}(G / P))^{T(G / P)}$ which upon twisting by $-T(G / P)$ yields a multiplication on $\operatorname{Hol}(G / P)^{-T(G / P)}$. Its associativity follows by arguing as in the proof of Theorem 1.1, where the lower right corner of a diagram analog to diagram (3-5) homotopy commutes because the map defining the homotopy associativity of $\operatorname{Rat}(G / P)$ is $P$-equivariant. Finally homotopy commutativity follows because the map defining homotopy commutativity of $\operatorname{Rat}(G / P)$ is $P$-equivariant.

\section{The loop spectral sequence and computations}

Our next objective is to determine the intersection product and module structure for some choices of $N$ and $M$. To that end we follow [8] (with minor adjustments) and introduce a graded module structure into the Serre spectral sequence for the evaluation fibration

$$
\operatorname{Map}^{*}(N, M) \longrightarrow \operatorname{Map}(N, M) \longrightarrow M
$$


so that on the $E_{\infty}$ level we obtain a graded version of the intersection module structure constructed in Section 3. The authors in [8] focused on the case $N=S^{1}$ and $M$ simply connected. In our case and in general $\operatorname{Map}(N, M)$ is not connected and so our construction ends up with an extra grading.

The basic case is when $N=S^{m}$. The following main calculational result is key to proving Theorem 1.5. It states in brief that by regrading suitably the Serre spectral sequence for (6-1) we end up with a multiplicative spectral sequence.

Proposition 6.1 Suppose that $M$ is a closed, compact, oriented, simply connected manifold, and assume $m \geq 1$. Then there is a spectral sequence of rings

$$
\left\{\mathbb{E}_{p, q}^{r}\left(S^{m}, M\right),-\operatorname{dim} M \leq p \leq 0, q \geq 0\right\},
$$

second quadrant in $p$ and $q$, and converging to the ring $\mathbb{H}_{*}\left(L^{m} M\right)$ in such a way as to have

$$
\mathbb{E}_{p, q}^{2} \cong H^{-p}\left(M, H_{q}\left(\Omega^{m} M\right)\right) .
$$

The product structure on $\mathbb{E}^{2}$ is given by the cup product in cohomology with coefficients in the Pontrjagin ring $H_{*}\left(\Omega^{m} M\right)$.

Proof As in [8], let $C_{*}\left(L^{m} M\right)$ be the singular chain complex of $L^{m} M$, where $C_{p}\left(L^{m} M\right)$ is the free abelian group on maps $\Delta^{p} \longrightarrow L^{m} M ; \Delta^{p}$ being the standard $p$-simplex, and consider the standard filtration of this chain complex by

$$
\{0\} \hookrightarrow \cdots \hookrightarrow F_{p-1} C_{*}\left(L^{m} M\right) \hookrightarrow F_{p} C_{*}\left(L^{m} M\right) \hookrightarrow \cdots \hookrightarrow C_{*}\left(L^{m} M\right)
$$

where $F_{p} C_{*}\left(L^{m} M\right)$ is the subchain complex generated by those $r$-simplices

$\tilde{\sigma}: \Delta^{r} \longrightarrow L^{m} M$ satisfying $e v \circ \tilde{\sigma}=\sigma\left(i_{0}, \ldots, i_{r}\right)$ for some $\sigma: \Delta^{q} \longrightarrow M$ and $\left(i_{0}, \ldots, i_{r}\right): \Delta^{r} \longrightarrow \Delta^{q}$ is a simplicial map sending the $k$-th vertex of $\Delta^{r}$ to the vertex $i_{k}$ of $\Delta^{q}$ with $\left.0 \leq i_{0} \leq \cdots \leq i_{r} \leq q\right)$, and $q \leq p$.

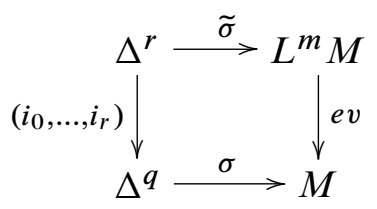

The spectral sequence arising from the above filtration is the Serre spectral sequence for the fibration $\Omega^{m} M \longrightarrow L^{m} M \longrightarrow M$. We now wish to show that the intersection product exists at the chain level

$$
c s_{*}: C_{i}\left(L^{m} M\right) \otimes C_{j}\left(L^{m} M\right) \longrightarrow C_{i+j-d}\left(L^{m} M\right)
$$


and is compatible with the filtrations as follows

$$
F_{p}\left(C_{*}\left(L^{m} M\right)\right) \otimes F_{q}\left(C_{*}\left(L^{m} M\right)\right) \longrightarrow F_{p+q-d}\left(C_{*}\left(L^{m} M\right)\right) .
$$

Similarly as in diagram (3-8) $c s_{*}$ is the composite of several chain maps.

$$
\begin{aligned}
& \times: C_{*}\left(L^{m} M\right) \otimes C_{*}\left(L^{m} M\right) \longrightarrow C_{*}\left(L^{m} M \times L^{m} M\right) \\
& c_{1}: C_{*}\left(L^{m} M \times L^{m} M\right) \longrightarrow C_{*}\left(\left(L^{m} M \times_{M} L^{m} M\right)^{\widetilde{v}}\right) \\
& c_{2}: C_{*}\left(\left(L^{m} M \times_{M} L^{m} M\right)^{\widetilde{v}}\right) \longrightarrow C_{*}\left(\left(L^{m} M\right)^{v}\right) \\
& w: C_{*}\left(\left(L^{m} M\right)^{v}\right) \longrightarrow C_{*}(D L, S L) \\
& \cap: C_{*}(D L, S L) \rightarrow C_{*-d}(D L) .
\end{aligned}
$$

Here $(D L, S L)$ is the pullback via evaluation $L^{m} M \rightarrow M$ of the pair $(D M, S M)$ corresponding to (disc,sphere) bundle of the tangent bundle of $M$. The first three maps are filtration preserving being induced from maps of spaces and $w$ is a filtration preserving weak equivalence. The map $w$ has the following explicit description. Choose a representative cochain $T_{M} \in C^{d}(D M, S M)$ for the Thom class of the tangent bundle which vanishes on degenerate simplices, and let $T_{L}=e v^{*}\left(T_{M}\right)$ be the corresponding Thom class of the pullback over $L^{m} M$. Excision shows that there is a chain equivalence $C_{*}(D L / S L, *) \longrightarrow C_{*}(D L, S L)$. Since by construction $\left(L^{m} M\right)^{v}=D L / S L$, the map $w$ is the composite

$$
C_{*}\left(\left(L^{m} M\right)^{v}\right)=C_{*}(D L / S L) \longrightarrow C_{*}(D L / S L, *) \longrightarrow C_{*}(D L, S L)
$$

The main assertion next is then to show that capping with $T_{L}$ decreases filtration by $d$ sending

$$
F_{p} C_{*}(D L) \stackrel{\cap T_{L}}{\longrightarrow} F_{p-d} C_{*}(D L)
$$

We use the argument given in [8, Theorem 8$]$ and correct in passing a slight mistatement there. Let $\widetilde{\sigma} \in F_{p}\left(C_{r} D L\right)$ be in filtration $p$ so that by definition $e v_{*}(\widetilde{\sigma})=\sigma\left(i_{0}, \ldots, i_{r}\right)$ (as in (6-2) with $L^{m} M$ replaced by $D L$ ), $i_{0} \leq \cdots \leq i_{r} \leq q \leq p$, and

$$
\begin{aligned}
e v_{*}\left(\widetilde{\sigma} \cap T_{L}\right)=e v_{*}(\widetilde{\sigma}) \cap T_{M} & =\sigma\left(i_{0}, \ldots, i_{r}\right) \cap T_{M} \\
& = \pm T_{M}\left(\sigma\left(i_{r-d}, \ldots, i_{r}\right)\right) \sigma\left(i_{0}, \ldots, i_{r-d}\right) .
\end{aligned}
$$

Since $T_{M}$ vanishes on degenerate simplices, this last expression is non-zero only if $i_{r-d}<\ldots<i_{r} \leq q$; that is when $i_{r-d} \leq q-d \leq p-d$. If we write $\left.(\sigma\rfloor_{q-d}\right)$ the restriction of $\sigma$ to its front $(q-d)$-face, then (6-4) shows that for $\tilde{\sigma} \in F_{p} C_{*}(D L)$

$$
\left.e v_{*}\left(\widetilde{\sigma} \cap T_{L}\right)=a(\sigma\rfloor_{q-d}\right)\left(i_{0}, \ldots, i_{r-d}\right)
$$


for some constant $a$, and hence by definition $\widetilde{\sigma} \cap T_{L} \in F_{p-d} C_{*}(D L)$ which establishes the claim, and (6-3).

To complete the proof we observe that since the intersection product is filtration decreasing (by $d$ ), it induces a pairing at every level of the Serre spectral sequence for $L^{m} M \longrightarrow M$

$$
\mu_{r}: E_{p, s}^{r} \otimes E_{q, t}^{r} \longrightarrow E_{p+q-d, s+t}^{r}
$$

When $M$ is simply connected, $E_{p, s}^{2}=H_{p}\left(M, H_{s}\left(\Omega^{m} M\right)\right)$ and $\mu_{2}$ takes the form

$$
H_{p}\left(M, H_{s}\left(\Omega^{m} M\right)\right) \otimes H_{q}\left(M, H_{t}\left(\Omega^{m} M\right)\right) \longrightarrow H_{p+q-d}\left(M, H_{s+t}\left(\Omega^{m} M\right)\right)
$$

which by construction corresponds to the intersection product with coefficients in the (commutative) Pontrjagin ring $H_{*}\left(\Omega^{m} M\right)$. Translating the spectral sequence $E^{r}$ to the left by $d$ as in $\mathbb{E}_{-s, t}^{r}:=E_{d-s, t}^{r}, s \geq 0$, yields a spectral sequence which now converges to the shifted homology groups $\mathbb{H}_{*}\left(L^{m} M\right)$. The spectral sequence converges because additively it is the Serre spectral sequence. Via Poincaré duality, the $E^{2}$-term becomes $H_{d-s}\left(M, H_{t}\left(\Omega^{m} M\right)\right) \cong H^{s}\left(M, H_{t}\left(\Omega^{m} M\right)\right)$ and the intersection product in homology gets replaced by the cup product. The spectral sequence is one of algebras with differentials behaving as derivations.

Remark 6.2 Two observations are in order:

(1) As the proof makes explicit, Proposition 6.1 is valid even for a disconnected fiber as is the case when $\Omega^{m} M$ has components. In this case there is an extra grading for the components, and this grading is additive under loop sum $\Omega^{m} M \times \Omega^{m} M \longrightarrow \Omega^{m} M$. The intersection product $\bullet$ can then be viewed as a"link" between the various components of the $L^{m} M$ and this is what we exploit most.

(2) Working with holomorphic mapping spaces, it is possible as above to introduce the intersection product directly in the spectral sequence computing the homology of $\operatorname{Hol}(n)$ and more generally $\operatorname{Hol}(G / P)$.

The proof of Proposition 6.1 used nothing special about $S^{m}$ aside from the fact that it is a co- $H$ space (namely in defining the map $c_{2}$ ). In fact a completely analogous statement can be made after replacing throughout $S^{m}$ by a coassociative co- $H$ space $S, \Omega^{m} M$ by $\operatorname{Map}^{*}(S, M)$ and $L^{m} M$ by $\operatorname{Map}(S, M)$.

This analogy can be taken up further. We can consider for a space $N$ the Serre spectral sequence for (6-1) as a second quadrant spectral sequence $\mathbb{E}(N, M)$ converging to the homology of $\operatorname{Map}(N, M)$ which by Poincaré duality for a simply connected $M$ can be written as

$$
\mathbb{E}_{p, q}^{2}(N, M)=H^{-p}\left(M, H_{q} \operatorname{Map}^{*}(N, M)\right),-d \leq p \leq 0, q \geq 0
$$


The following theorem admits a proof completely analogous to that of Proposition 6.1.

Theorem 6.3 Let $S$ be a coassociative co- $H$ space coacting on a space $N$. Then $\mathbb{E}^{r}(N, M)$ is a differential graded module over $\mathbb{E}^{r}(S, M)$, with action

$$
\mathbb{E}_{p, s}^{r}(S, M) \otimes \mathbb{E}_{q, t}^{r}(N, M) \longrightarrow \mathbb{E}_{p+q, s+t}^{r}(N, M)
$$

When $r=2$, the action corresponds to the map obtained via cup product on $H^{*}(M)$ and the $H_{*}\left(\operatorname{Map}^{*}(S, M)\right)$-module structure of $H_{*}\left(\operatorname{Map}^{*}(N, M)\right)$ induced by the coaction map $N \longrightarrow N \vee S$. Moreover the module structure of the $E^{\infty}$-terms is compatible with the $\mathbb{H}_{*}(\operatorname{Map}(S, M))$-module structure of $\mathbb{U}_{*}(\operatorname{Map}(N, M))$.

The above theorem is particularly useful to us when $N$ is a closed oriented $m$-manifold, $S$ is the $m$-sphere and $N \longrightarrow N \vee S$ the pinch map.

\subsection{Applications}

We are now in a position to prove theorems 1.5 and 1.8 .

Proof of Theorem 1.5 Apply Proposition 6.1 to the case $m=2, M=\mathbb{P}^{n}$. Write $\mathbb{H}_{i}=H_{2 n+i}$, and grade negatively the cohomology ring $H^{*}\left(\mathbb{P}^{n}\right)=\mathbb{Z}_{2}[c] / c^{n+1}$, $c \in H^{-2}\left(\mathbb{P}^{n}\right)$. Recall that $\iota \in H_{0}\left(\Omega_{1}^{2} \mathbb{P}^{n}\right)$ and $u \in H_{2 n-1}\left(\Omega_{1}^{2} \mathbb{P}^{n}\right)$ are the generators coming from the inclusion $S^{2 n-1} \simeq \operatorname{Rat}_{1} \mathbb{P}^{n} \hookrightarrow \Omega_{1}^{2} \mathbb{P}^{n}$.

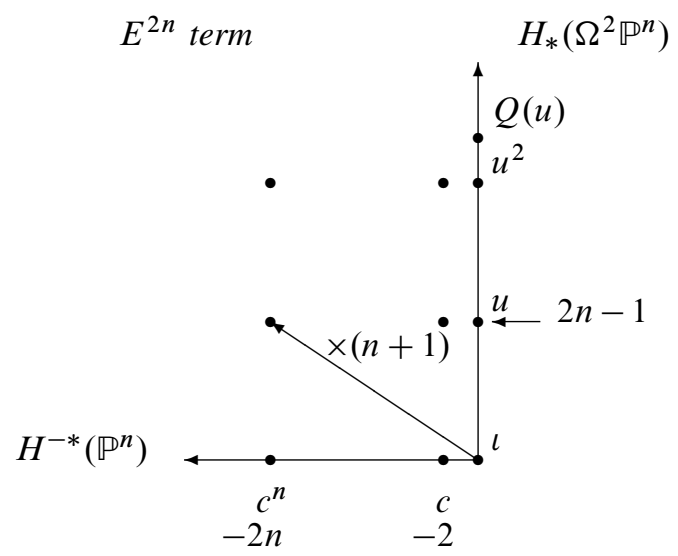

The String Spectral Sequence Mod 2 for $L^{2} \mathbb{P}^{n}$

The second quadrant spectral sequence associated to $\Omega^{2} \mathbb{P}^{n} \longrightarrow L^{2} \mathbb{P}^{n} \longrightarrow \mathbb{P}^{n}$ (Proposition 6.1) has as $E^{2}$ term the algebra $E_{*, *}^{2}=H^{*}\left(\mathbb{P}^{n}\right) \otimes H_{*}\left(\Omega^{2} \mathbb{P}^{n}\right)$ and converges to $\mathbb{H}_{*}\left(L^{2} \mathbb{P}^{n}\right)$. For dimensional reasons $E^{2 n+1}=E^{\infty}$. 
According to Proposition 2.2, in the spectral sequence for $\mathrm{Hol}_{1}(n)$ there is a differential from the fundamental class $\left[\mathbb{P}^{n}\right]$ in the base to the spherical class $u$ in the fiber. By comparison we get the same differential for $\Omega_{1}^{2} \mathbb{P}^{n} \rightarrow L_{1}^{2} \mathbb{P}^{n} \rightarrow \mathbb{P}^{n}$. Going to the spectral sequence for $\mathbb{H}_{*}\left(L^{2} \mathbb{P}^{n}\right)$, the class $\left[\mathbb{P}^{n}\right]$ translates to $\iota \in E_{0,0}^{2}$, while $u$ translates to $c^{n} u \in E_{-2 n, 2 n-1}^{2}$. The Euler class differential in Lemma 2.1 translates in turn to the differential $d_{2 n} \iota=(n+1) c^{n} u$. Note that the fact that $d$ is a derivation implies that for any integer $k$

$$
d_{2 n} \iota^{k}=(n+1) k c^{n} u \iota^{k-1}
$$

This recovers the differential from Proposition 2.2, and also settles the rational case (eg Corollary 2.3) since $\Omega^{2} \mathbb{P}^{n}$ is rationally $\mathbb{Z} \times S^{2 n-1}$, with rational homology $\mathbb{Q}\left[\iota, \iota^{-1}\right] \otimes$ $E[u]$.

With mod-2 coefficients, there are additional generators $Q^{i}(u)$ in $H_{*}\left(\Omega_{2^{i}}^{2}\left(\mathbb{P}^{n}\right) ; \mathbb{Z}_{2}\right)$. But $Q^{i}(u)$ is the top class in $H_{*}\left(\operatorname{Rat}_{2^{i}}(n) ; \mathbb{Z}_{2}\right)$ and hence by comparison $d_{2 n} Q^{i}(u)=0$ in the spectral sequence for the continuous mapping space.

For $p$ an odd prime, the procedure is analogous, for $Q^{i}(u)$ is the top class in the homology mod $p$ of $\operatorname{Rat}_{p^{i}}(n)$, and its Bockstein is the image of the top class of the same space with coefficients in $\mathbb{Z}_{(p)}$.

The homology of $\operatorname{Hol}(n)$ is obtained by comparison and injects into the homology of $L^{2}\left(\mathbb{P}^{n}\right)$; the $E^{2 n}$ term for $\operatorname{Hol}(n)$ being a direct summand of the $E^{2 n}$ term for $L^{2}\left(\mathbb{P}^{n}\right)$. This proves the second part of the theorem.

By means of Theorem 1.5 one easily deduces Corollary 1.7 in the introduction. We work out one example to illustrate how the calculations go.

Example 6.4 We determine all differentials in the Serre spectral sequence $E^{r}$ mod 2 for the fibration $\Omega^{2} \mathbb{P}^{n} \rightarrow L^{2} \mathbb{P}^{n} \stackrel{e v}{\longrightarrow} \mathbb{P}^{n}$.

For $n$ odd the spectral sequence collapses at the $E^{2}=E^{2 n}$ term because $d_{2 n}$ is trivial on all multiplicative generators: $c, \iota, u$ and $Q^{i}(u)$, for $i>0$.

For $n$ even and again according to (6-5), the differential $d_{2 n}$ acts on a basis element of the form $\iota^{p} u^{q} Q$, where $Q$ is a product of iterated Dyer-Lashof operations $Q^{i}(u)$, by $d_{2 n}\left(\iota^{p} u^{q} Q\right)=p \iota^{p-1} u^{q+1} Q c^{n}$. Thus the surviving basis elements in the column 0 have the form $\iota^{p} u^{q} Q$ with $p$ even, and those in the column $-2 n$ have the form $\iota^{p} u^{q} Q c^{n}$ with $p$ odd. If

$$
A=\mathbb{F}_{2}\left[\iota^{2}, \iota^{-2}, u\right] \otimes \mathbb{F}_{2}\left[Q^{i}(u), i>0\right] \subset H_{*}\left(\Omega^{2} \mathbb{P}^{n} ; \mathbb{F}_{2}\right)
$$


then for $n$ even we have the additive isomorphism

$$
H_{*+2 n}\left(L^{2} \mathbb{P}^{n} ; \mathbb{F}_{2}\right)=A \oplus c H_{*}\left(\Omega^{2} \mathbb{P}^{n} ; \mathbb{F}_{2}\right) \oplus \cdots \oplus c^{n-1} H_{*}\left(\Omega^{2} \mathbb{P}^{n} ; \mathbb{F}_{2}\right) \oplus \iota c^{n} A .
$$

Let now $N=C$ be a compact Riemann surface (of fixed genus) and $M=\mathbb{P}^{n}$.

Proof of Theorem 1.8 Since the components of $\operatorname{Map}\left(C, \mathbb{P}^{n}\right)$ are indexed by the integers, we introduce in the spectral sequence $\mathbb{E}\left(C, \mathbb{P}^{n}\right)$ of Theorem 6.3 a trigrading as in Proposition 6.1. This spectral sequence converges to $\bigoplus_{k \in \mathbb{Z}} \mathbb{H}_{*}\left(\operatorname{Map}_{k}\left(C, \mathbb{P}^{n}\right)\right)$. The differential on $\iota^{k}$ in $\mathbb{E}_{0,0, k}^{2 k}\left(S^{2}, \mathbb{P}^{n}\right)$ is the class $(n+1) k u \iota^{k-1} c^{n}$ which is trivial when $p$ divides $k(n+1)$. This implies in that case that $\iota^{k}$ survives to the $\mathbb{E}^{\infty}$-term and it is invertible with inverse $\iota^{-k}$. Multiplication by $\iota^{k}$ switches up components by $k$ as in

$$
\mathbb{H}_{*}\left(\operatorname{Map}_{i}\left(C, \mathbb{P}^{n}\right) ; \mathbb{Z}_{p}\right) \stackrel{\iota^{k}}{\longrightarrow} \mathbb{H}_{*}\left(\operatorname{Map}_{i+k}\left(C, \mathbb{P}^{n}\right) ; \mathbb{Z}_{p}\right)
$$

and hence yields an isomorphism.

\section{References}

[1] C P Boyer, B M Mann, J C Hurtubise, R J Milgram, The topology of the space of rational maps into generalized flag manifolds, Acta Math. 173 (1994) 61-101 MR1294670

[2] M Chas, D Sullivan, String topology arXiv:math.GT/9911159

[3] D Chataur, A bordism approach to string topology, Int. Math. Res. Not. (2005) 28292875 MR2180465

[4] F R Cohen, R L Cohen, B M Mann, R J Milgram, The topology of rational functions and divisors of surfaces, Acta Math. 166 (1991) 163-221 MR1097023

[5] R L Cohen, Multiplicative properties of Atiyah duality, Homology Homotopy Appl. 6 (2004) 269-281 MR2076004

[6] R L Cohen, V Godin, A polarized view of string topology, from: "Topology, geometry and quantum field theory", London Math. Soc. Lecture Note Ser. 308, Cambridge Univ. Press, Cambridge (2004) 127-154 MR2079373

[7] R L Cohen, J D S Jones, A homotopy theoretic realization of string topology, Math. Ann. 324 (2002) 773-798 MR1942249

[8] R L Cohen, J D S Jones, J Yan, The loop homology algebra of spheres and projective spaces, from: "Categorical decomposition techniques in algebraic topology (Isle of Skye, 2001)”, Progr. Math. 215, Birkhäuser, Basel (2004) 77-92 MR2039760

[9] Y Félix, L Menichi, J-C Thomas, Gerstenhaber duality in Hochschild cohomology, J. Pure Appl. Algebra 199 (2005) 43-59 MR2134291 
[10] J Gravesen, On the topology of spaces of holomorphic maps, Acta Math. 162 (1989) 247-286 MR989398

[11] K Gruher, P Salvatore, String topology in a general fiberwise setting and applications arXiv:math.AT/0602210

[12] J W Havlicek, The cohomology of holomorphic self-maps of the Riemann sphere, Math. Z. 218 (1995) 179-190 MR1318152

[13] P Hu, Higher string topology on general spaces arXiv:math. AT/0401081

[14] J C Hurtubise, Configurations de particules et espaces de modules, Canad. Math. Bull. 38 (1995) 66-79 MR1319902

[15] S Kallel, Configuration spaces and the topology of curves in projective space, from: "Topology, geometry, and algebra: interactions and new directions (Stanford, CA, 1999)", Contemp. Math. 279, Amer. Math. Soc., Providence, RI (2001) 151-175 MR1850746

[16] S Kallel, P Salvatore, work in progress

[17] J R Klein, Fiber products, Poincaré duality and $A_{\infty}$-ring spectra, Proc. Amer. Math. Soc. 134 (2006) 1825-1833 MR2207500

[18] A W Knapp, Lie groups beyond an introduction, Progress in Mathematics 140, Birkhäuser, Boston (1996) MR1399083

[19] J Leborgne, String Spectral Sequences arXiv:math. AT/0409597

[20] G Segal, The topology of spaces of rational functions, Acta Math. 143 (1979) 39-72 MR533892

[21] R M Switzer, Algebraic topology-homotopy and homology, Classics in Mathematics, Springer, Berlin (2002) MR1886843Reprint of the 1975 original

Laboratoire Painlevé, Université de Lille I

Villeneuve d'Ascq, France

Dipartimento di matematica, Università di Roma "Tor Vergata"

Roma, Italy

sadok.kallel@math.univ-lille1.fr, salvator@mat.uniroma2.it

Proposed: Ralph Cohen

Seconded: Haynes Miller, Bill Dwyer
Received: 23 September 2003

Revised: 28 August 2006 